\title{
Comparative study between L-carnitine versus co-enzyme Q10 as an adjuvant therapy to clomiphene resistant pcos women on improving pregnancy rate and prevention of early pregnancy loss
}

Original Article
Safaa Ibrahim

Department of Obstetrics and and Gynecology, Cairo University, Cairo, Egypt

\begin{abstract}
Aim of the work: Compare the effectiveness of L-carnitine versus Co-enzyme Q10 on improving pregnancy rates and decraese the rate of early pregnancy loss in patients with PCO resistant to clomiphene citrate.

Setting and Design: A prospective randomized controlled study conducted in infertility clinic of a tertiary care center from June 2017 to June 2019.

Patients and Methods: A total of 225 subjects presenting with infertility and diagnosed with PCOS (based on Rotterdam criteria 2003) and resistant to clomiphene citrate were enrolled in the study. Subjects were randomly allocated into three groups. Group A: received clomiphene citrate (CC) only, Group B: received clomiphene citrate plus L-carnitine (2gm). Group C: received clomiphene citrate plus co- enzyme Q10 (200 mg). Main outcome measures include the ovulation rate, cumulative clinical pregnancy rate (CPR) are the primary outcome measures.

Results: The ovulation rate was significantly higher among the groups B,C compared to group A (62.5\%, $66.6 \%$ vs $18.3 \%$ respectively). Total number of cases who get pregnant were higher in Group B 35(47.3\%), Group C 33(44.6\%) compared to Group A 6(8.1\%). Cumulative Clinical pregnancy rate was 3\%, 49.3\%, and 47.8\% for Group A, Group B, and Group $\mathrm{C}$, respectively. The abortion rate was low in Group B $4 \%$ and significantly lower in Group C 2.7\% compared to Group A $6.7 \%$.

Conclusion: Adding either L-carnitine or Co-enzyme Q10 to clomiphene citrate in patients with clomiphene-resistant PCOS were beneficial in increasing the quality of ovulation, the clinical pregnancy rate salso in decreasing the rate of early pregnancy loss.
\end{abstract}

Key Words: Co-enzyme Q10, induction of ovulation, infertility, L-Carnitine, PCOS.

Received: 16 December 2019, Accepted: 13 January 2020

Corresponding Author: Safaa Ibrahim, Department of Obstetrics and Gynecology, Cairo University, Cairo, Egypt, Tel.: 01012890590 , E-mail: safysafy1974@gmail.com

ISSN: 2090-7265, May 2020, Vol.10, No.2

\section{INTRODUCTION}

PCO is one of the most common endocrine disorders affecting $7-15 \%$ of women in their child bearing age $^{[1,2]}$. The clinical manifestations of the disease mainly oligomenorrhea, amenorrhea, ovulatory dysfunction, hirsutism, obesity.

The impact of that syndrome on human reproduction does not only include anovulatory infertility but also detrimental effects on oocyte, embryo quality endometrial receptivity and pregnancy development ${ }^{[3]}$.

Ovulatory dysfunction accounts for 30 to $40 \%$ of infertility in women ${ }^{[4]}$. In individuals with ovulatory dysfunctions and particularly in those suffering from Polycystic Ovarian Syndrome (PCO), ovulation induction is achieved through various regimens. However, Conventional dosages do not yield adequate results in some patients with $\mathrm{PCO}$; while some of them are, indeed, responsive to increased dosages ${ }^{[5]}$.

Women who have PCO do appear to be at higher risk for having miscarriage (the rate of early stage abortion is $35-50 \%$ in pregnant PCOS patients after ovulation induction $)^{[6,7]}$. Though the cause for this relationship is unclear, researchers believe that few factors may be to belame. First, women with PCOs tend to have longer menstrual cycles (late ovulation), this exposes the developing egg to lots of hormones, possibly damaging $\mathrm{it}^{[8,9]}$.

Second, there is known relationship between obesity, high androgen levels, hyperinsulinemia and insulin resistance that may contribute to poor egg quality, endometrial dysfunction, meaning problem of implantation and increased risk of early miscarriage ${ }^{[9,10]}$. Also, repeated ovulation induction decreases the quantity of mitochondrial 
DNA in oocytes that lead to poor egg quality, in addition the ovulated oocytes number declines markedly with repeated cycles of ovulation ${ }^{[11,12]}$.

Anti estrogen medicines such as Clomiphene Citrate has been used for ovulation induction since 1960s and used in the first round of treatment for PCOS patientswho wish to conceive in usual doses $50-150 \mathrm{mg}^{[13]}$. The major application of Clomiphene is in women who suffer from infertility caused by normogonadotropic, normoestrogenic, and normoprolactinemic anovulation (WHO type II anovulatory infertility), which also include $\operatorname{PCOS}^{[14]}$.

If patients fail in ovulation with a maximun dosing of $250 \mathrm{mg}$ of Clomiphene or if the patients does not demonstrate a proper response after at least three cycles of treatment with usual doses of Clomiphene (in the absence of other infertility factors), this defines clomiphene resistance which occur in approximately 15 to $40 \%$ of PCOS women ${ }^{[15]}$, other ovarian stimulation methods and techniques are employed such as gonadotrophins induction, adding metformin, N-acetylcysteine, or doing surgical intervention such as laparoscopic ovarian drilling ${ }^{[16]}$.

Adding L-Carnintine or Co-enzyme Q10 to clomiphene citrate could potentially result in desirable follicles count,with the same conventional dosage ${ }^{[17]}$.

L-Carnitine is a small water-soluble molecule that have immense functional capabilities to regulate the oxidative and metabolic status of the female reproductive system. It palys an important role in fat metabolism. It also plays a fundamental role in the normal mitochondrial oxidation of fatty acids and generation of Acyl-CoA esters, which increase the supply of energy to the cells. L-Carnitine also prevents damage caused by oxygen free radicals to the membrane and $\mathrm{DNA}^{[18,19]}$.

L-Carnitine is responsible for neutralization of free radicals, removal of superoxide anions, and inhibition of lipid preoxidation. Therefore, L-Carnitine is capable of preventing hydrogen peroxide damages,can stabilize organelle and protect the cell from apoptosis ${ }^{[20]}$.

Some recent studies found that women with PCO had lower level of serum L-carnitine that was correlated to hyper androgenic and hyper insulinemic states. Hence, that adding LC may help to reverse the metabolic disorders associated with PCOS and obesity that can be reversed by carnitine $^{[21]}$.

The previous study of AM. Ismail et al showed that the combination of L-carnitine and clomiphene citrate significantly improved both the ovulation and the pregnancy rates in clomiphene resistant PCOS (64.7\% vs. $17.6 \%$ and $51.25 \%$ vs. $5.8 \%)^{[22]}$

Coenzyme Q 10 is a fat-soluble vitamin like substance with an antioxidant effect, found naturally in each cell of our body particularly prevalent in mitochondria. It plays a key role in energy production. Coenzyme Q10 is a component of the electron transport chain responsible for generating energy.

Originally known for its benefits to cardiac and neurological health but now has been the subject of considerable research into its effect on the oocytes and enhancing female fertility and improving ovulation espicially in PCO womenbeing lipid-soluble antioxidant thatcould reduce oxidation stress within the ovary protect DNA from free radical induced oxidative damage ${ }^{[23]}$.

According to previously published studies: decreased energy production in oocytes with aging may be related to a deficiency of CoQ10 which is a micronutrient, and the extent of tissue uptake of CoQ10 correlates with the degree of tissue deficiency. Additionally, with time, eggs are exposed to more damagingoxidative stress due to free radicals. As an antioxidant, $\mathrm{CoQ} 10$ has been linked to improving egg quality and ovulation by repairing free radicals ${ }^{[24,25]}$

Co Q10 can regenerate vitamin E from its oxidized form, this interaction with vitamin $\mathrm{E}$ is important for the lipoproteins protection gainst oxidative damage ${ }^{[26]}$. Finally, it acts as antiapoptotic, and it is well known that apoptosis is the main mechanism involved in follicularcohort atresia.

Co-enzyme Q10 (Co Q10) is often recommended by reproductive endocrinologists to help improve egg/embryo quality for women who may be classed as poor responders with poor ovarian reserve. But, Co Q10 may have more diverse uses in female reproductive medicine than was previously thought, it may help women with PCOS to conceive more successfully with clomiphene (Clomid).

New research has showed that Co Q10 and clomiphene citrate in the treatment of clomiphene-citrate-resistant PCOS patients improves ovulation and pregnancy ratesA prospective randomized controlled studyincluded total of 101 infertile women with PCOS that were randomized either to combined Co Q10 and clomiphene citrate or to clomiphene citrate alone. Those who supplemented with Co Q10 saw more follicles and greater endometrial thickness. Ovulation occurred in $65.9 \%$ in the Co Q10 group compared with only $15.5 \%$ in the control group. Pregnancy rate was significantly higher in the Co Q10 group $(37.3 \%)$ versus the control group $(6.0 \%)^{[27]}$.

In $\mathrm{PCO}$ the high energy consuming processes associated with fertilization and embryo development will be supplemented by adding L Carnitine and CO Q10 resulting in improving both egg quality and subsequent fertilization rates.

\section{PATIENTS AND METHODS}

\section{Outcome Measures \\ Primary outcomes}

The main outcome measures of the study include: 
1- Ovulation rate.

2-Cumulative Cinical pregnancy rate (Clinical pregnancy is defined as ultrasound confirmation of gestational sac with fetal cardiac activity).

\section{Secondary outcome measures}

1-Abortion rate include biochemical pregnancies (cases with +ve pregnancy test and abort spontaneously before reaching the stage of clinical pregnancy) + cases who abort before 12 week of pregnancy.

2- Multiple pregnancy.

This is a prospective parallel randomized control study in a 1:1 allocation ratio conducted in infertility clinic of a tertiary care centerfrom June 2017 to June 2019. The study included women attending the infertility clinicdiagnosed with PCOS with no other abnormalities. This diagnosis based on European Society for Human Reproduction and Embryology/American Society for Reproductive Medicine (ESHRE/ASRM) guidelines criteria (Rotterdam criteria 2003)

\section{Inclusion Criteria}

The study included:1- patients with history of infertility (primary or secondary) diagnosed with PCO as the presence of two of three features (oligo and/or anovulation, clinical and/or biochemical signs of hyperandrogenism, and polycystic ovary): Rotterdam criteria 2003.

2- Patients with ovulatory cause of infertility only (i.e. Lack of other infertility factors such as the tubal or male factor. The diagnosis was based on obtaining a complete history, a physical examination and a documented complete infertility work-up within the previous six months, either conducted within the setting of the hospital or at a licensed infertility management clinic

3-Patients who are resistence to Clomiphene citrate who had received (3- 6 unsuccessful $150 \mathrm{mg}$ clomiphene citrate stimulation cycles) and failed to have a follicular response

4- Patients aged between 20 and 35 years and BMI 18-30 are included

5-Serum FSH, LH, estradiol measured on day 3 of cycle (basal) are varied in the normal range in patients who had received clomiphene citrate but showed no response.

6- Patients with normal prolactin and TSH levels are included.

\section{Exclusion Criteria}

The exclusion criteria for this research included

1- Sensitivity to L - Carnitine or Co-enzyme Q 10 and lack of interest in participating in the research.

2- Age more than 35 years.

3- Tubal, uterine or male factor of infertility.

4- FSH exceeding $10 \mathrm{IU} / \mathrm{ml}$ are excluded.

Calculation of the sample size was based on the primary outcomes, clinical pregnancy rate and abortion rate. Depending on the results of a previous study of AM. Ismail et al who compared clomiphene citrate plus L-carnitine versus clomiphene citrate plus placebo, pregnancy rate was $51.5 \%$ vs. $5.8 \%$ respectively ${ }^{[19]}$. Considering the abortion rate in the same study of AM. Ismail and his colleagues was $4.7 \%$ vs. $2.4 \%$ in the placebo and L-carnitine groups respectively, and assuming the aborion rate in the coenzyme Q10 group will be $2.5 \%$. Accordingly, the calculated minimum optimum sample size was 65 women in each group. Adding 15\% of cases for the expected dropped out patients before the end of the study so each group will include 75 cases.Sample sizecalculation was done based on comparing 3 proportions from independent samples using Chi test, the $\alpha$ - error level was fixed at 0.05 , the power was set at $99 \%$ and the intervention groups ratio was set at 1 . calculation was done using $G^{*}$ Power software version 3.1.2 for MS Windows, Franz Faul, Kiel University, Germany.

Accordingly, the study included 225 cases that were eligible to share in the study. After explaining about the drugs used to induce ovulation and the necessity of regular frequent visits for follicular monitoring, they were randomly allocated (via computer-generated randomization) into 3 Groups:

- Group A: received 150 mg/day Clomiphene Citrate (CC) only.

- Group B received CC $150 \mathrm{mg}$ /day plus oral L-carnitine (2 g) daily.

- Group C receive CC $150 \mathrm{mg} /$ day plus Co- enzyme Q10 (200 mg) daily.

All subjects received oral $\mathrm{CC}$ from day three until day seven of the cycle. Both L-carnitine and Co-enzyme Q10taken from day three until the day of the first positive pregnancy test.

Informed, written consent taken from the participants prior to the study, they have the right to refuse to participate and/or withdraw from the study at any time.

All of the possible side effects of this treatment as nausea, vomiting, headache, diarrhoea, and sleep problem were explained to the paricipants before taking part in this research. However, these side effects have been reported to be rare in occurance.

All participants that give a written informed consent underwent a detailed history taking and thorough clinical examination including general physical examination, systemic, abdominal and gynecological examination. Baseline investigations and hormonal profile (serum folliclestimulating. Serum follicle-stimulating hormone (FSH), luteinizing hormone (LH), Thyroid stimulating hormone (TSH), Prolactin(PRL), Free Testosterone and Estradiol E2 concentrations on day three (basal) will be measured and patients with FSH values equal to or exceeding $10 \mathrm{mIU} / \mathrm{mL}$ are excluded. Serum estradiol measured in the same day of $\mathrm{HCG}$ injection. progesterone $(\mathrm{ng} / \mathrm{mL})$ will be measured on day 8 post HCG. Transvaginal ultrasonographic follicular monitoring is performed on all patients on days seven and 
nine and then individualized according to response. Size and number of dominant follicles seen in both ovaries and the endometrial thickness are estimated by ultrasound. When one leading follicle attained a diameter of $17 \mathrm{~mm}$ or more, 10,000 IU of hCG will be given (im injection). Timed intercourse advised starting every other day for one week 24-36 hours after hCG administration. Progesterone luteal-phase support will be given in all groups.

$\beta$-hCG was checked if the patient experienced one week missed period. Moreover, clinical pregnancy was confirmed by the presence of intrauterine gestational sac with a positive fetal heart beats viewed by transvaginal sonography 2-3 weeks after a positive pregnancy test. Biochemical pregnancy is defined as falling $\beta$-hCG concentration on serial determination.

Patients with positive pregnancy test were followed up till 12 weeks of pregnancy.

The induction of ovulation was repeated for 3 successive cycles if pregnancy was not achieved.

The primary outcomes were the ovulation rate, cummulative clinical pregnancy rate documented after 3 months of induction.

\section{Statistical Analysis}

Data were statistically described in terms of mean \pm standard deviation $( \pm \mathrm{SD})$, median and range, or frequencies (number of cases) and percentages when appropriate. Comparison of numerical variables between the study groups was done using one way analysis of variance (ANOVA) test. For comparing categorical data, Chi-square $\left(x^{2}\right)$ test was performed. Exact test was used instead when the expected frequency is less than 5. Two sided $p$ values less than 0.05 was considered statistically significant. All statistical calculations were done using computer program IBM SPSS (Statistical Package for the Social Science; IBM Corp, Armonk, NY, USA) release 22 for Microsoft Windows.

\section{RESULTS}

A total 248 women with primary or secondary infertility and diagnosed to have PCOS were screened regarding eligibility for the study, twenty three women excluded as they did not meet the inclusion criteria and refused to participate.

The 225 women eligible to participate were randomly allocated (1:1:1) to 3 groups Group A (control $n=75$ ), Group B (L Carnitine group $\mathrm{n}=75$ ), Group C (Co Q 10 group n75). Three patients withdrew after randomization without receiving their allocated medicineone from group A and two cases from group C. (withdrew their consent, did not come at scheduled time), ten patients were excluded (dropped out) after receiving their allocated medicine, three women from group A, three women from group B and four patients from group $\mathrm{C}$ as follows: excessive number of follicles, non rupture of the dominant follicle, and lost to follow up (Fig 1).

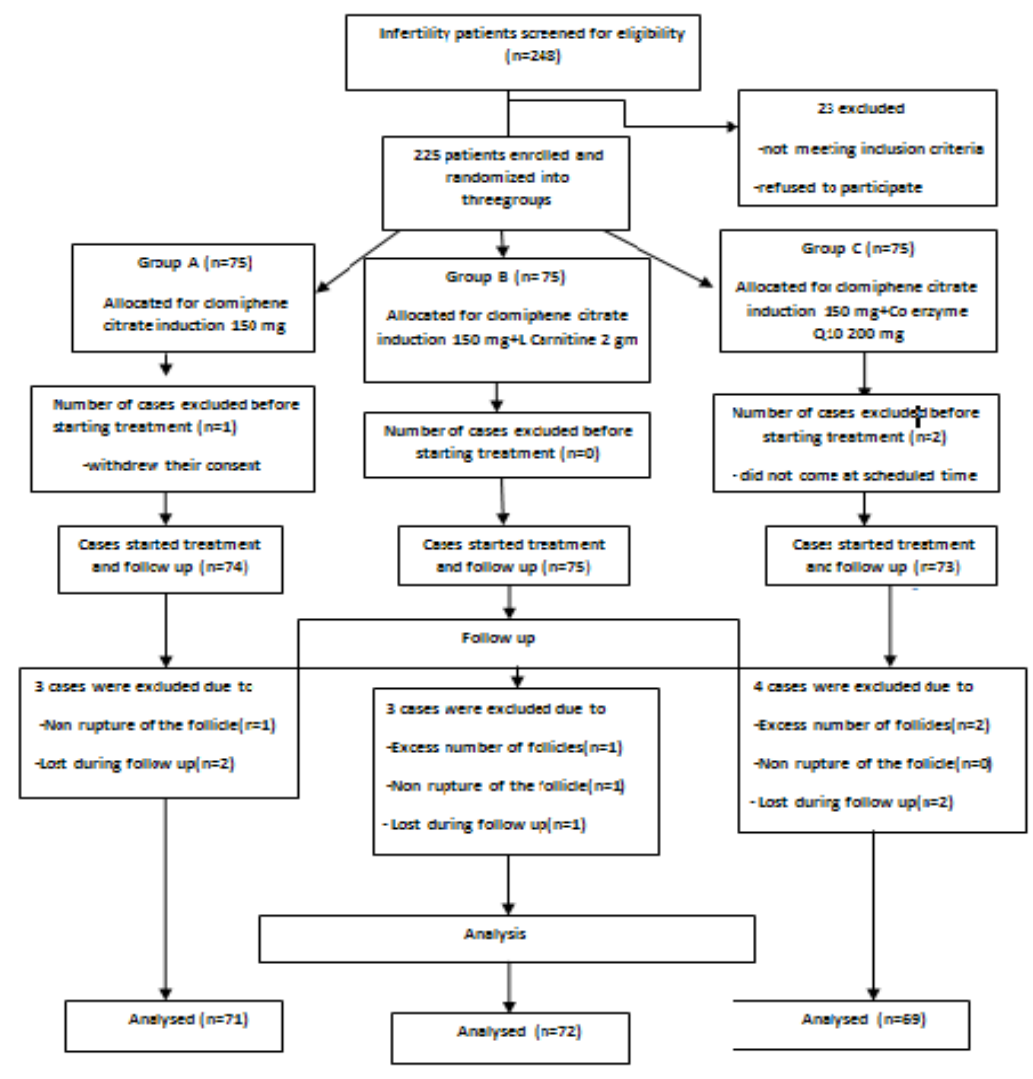

Fig. 1: Flow chart showing analysis of the study groups. 
The baseline characteristics of the three groups, such as age, duration of infertility, type of infertility, body mass index and basal hormonal levels were not statistically different. The mean age of the patients among the groups was 27.6 \pm 4.6 years while the mean BMI was 25.3 \pm 3.5 . Duration of infertility ranged from 2-6 years in all groups. The majority of cases had primary infertility $48 / 64 \%, 52 / 69.3 \%, 46 / 61.3 \%$ in group A, B, C respectively. (Table 1).

Table 1: Demographic Data and Hormonal profile of the study cases; Baseline characteristics of the patients in the three study groups

\begin{tabular}{|c|c|c|c|c|}
\hline & $\begin{array}{c}\text { Group A } \\
\text { (Control) }\end{array}$ & Group B & Group C & $P$ value \\
\hline $\mathrm{N}^{*}$ & $(\mathrm{n}=71)$ & $(\mathrm{n}=72)$ & $(\mathrm{n}=69)$ & \\
\hline Age (years) & $27.7 \pm 4.7$ & $27.7 \pm 4.6$ & $27.3 \pm 4.6$ & 0.858 \\
\hline Infertility duration (years)* & $3.8 \pm 1.3$ & $3.7 \pm 1.3$ & $4.2 \pm 1.2$ & 0.593 \\
\hline BMI $(\mathrm{kg} / \mathrm{m} 2)^{*}$ & $25.6 \pm 3.45$ & $25.7 \pm 3.36$ & $24.5 \pm 3.68$ & 0.593 \\
\hline \multicolumn{5}{|l|}{ Infertility type** } \\
\hline Primary $(\%)$ & $48(64 \%)$ & $52(69.3 \%)$ & $46(61.3 \%)$ & \\
\hline Secondary $(\%)$ & $27(36 \%)$ & $23(30.7 \%)$ & $29(38.7 \%)$ & 0.579 \\
\hline Mean no.of failed clomipnene induced cycles* & $5.2 \pm 1.4$ & $4.88 \pm 0.99$ & $4.9 \pm 0.93$ & 0.114 \\
\hline Day 3 FSH (mIU/mL)* & $5.2 \pm 1.06$ & $5.5 \pm 0.96$ & $5.39 \pm 1.03$ & 0.323 \\
\hline Day 3 LH (mIU/mL)* & $10.7 \pm 1.64$ & $10.9 \pm 1.59$ & $10.6 \pm 1.39$ & 0.418 \\
\hline Day 3 E2 (pg/ml)* & $77.5 \pm 12.15$ & $77.7 \pm 12.52$ & $81.3 \pm 13.72$ & 0.134 \\
\hline Day 3* F.testosterone(ng/dl) & $3.15 \pm 1.18$ & $3.08 \pm 1.25$ & $3.19 \pm 1.13$ & 0.849 \\
\hline TSH (mIU/L)* & $2.5 \pm 1.12$ & $2.4 \pm 1.1$ & $2.3 \pm 1.13$ & 0.530 \\
\hline $\operatorname{PRL}(\mathrm{ng} / \mathrm{ml}) *$ & $16.19 \pm 5.1$ & $15.17 \pm 5.5$ & $15.9 \pm 5.1$ & 0.458 \\
\hline
\end{tabular}

$\mathrm{N}=$ number $\quad *$ Data represent means \pm standard deviation BMI: Body Mass Index $\quad * *$ Data represent number (percentage).

There was highly significant difference in the meanof number of follicles $>17 \mathrm{~mm}$, follicle size, day of $\mathrm{HCG}$ trigger between the control and study groups. The mean number of follicles $>17 \mathrm{~mm}$ was in Group A $(0.21 \pm 0.47)$ compared to Goup B was ( $0.93 \pm 0.89)$ and in Group C was $(1.19 \pm 1.06)$. The follicle size was signficantly larger in Group B and C compared to Group A $(18.18,18.45$ vs 17.34 respectively, $p$ value $(<0.0001)$. HCG was given earlier in Group C and Group B compared to Group A.
Also there was significant difference in endometrial thickness (millimetre) measured on the day of the trigger between the control and study groups, it is ranged between $5-8 \mathrm{~mm}$ in group $\mathrm{A}$, while in group B it ranged between $7-10 \mathrm{~mm}$ and in group $\mathrm{C}$ ranged between $8-11$ $\mathrm{mm}(p<0.0001)$. E2 level on day of HCG trigger and progesterone level on day 8 post $\mathrm{HCG}$ noticed to be higher in Group $\mathrm{C}$ and Group $\mathrm{B}$ than in Group A (Table 2). 
Table 2: The cycle characteristics of the groups:

\begin{tabular}{|c|c|c|c|c|}
\hline \multirow{2}{*}{ Parameter } & Group (A) & Group (B) & Group (C) & \multirow{2}{*}{$P$-value } \\
\hline & $(\mathrm{n}=71)$ & $(\mathrm{n}=72)$ & $(n=69)$ & \\
\hline Mean number of dominant follicles $>17 \mathrm{~mm}$ & $0.21 \pm 0.47$ & $0.93 \pm 0.89$ & $1.19 \pm 1.06$ & $0.0001 *$ \\
\hline Mean follicle size $(\mathrm{mm})$ on the day of trigger & $17.34 \pm 0.39$ & $18.18 \pm 0.66$ & $18.45 \pm 0.74$ & $0.0001 *$ \\
\hline Day of HCG trigger & $15.25 \pm 1.13$ & $11.37 \pm 1.08$ & $10.93 \pm 1.18$ & $0.0001^{*}$ \\
\hline Mean endometrial thickness on the trigger day & $6.1 \pm 0.71$ & $8.5 \pm 0.76$ & $8.8 \pm 0.87$ & $0.000^{*}$ \\
\hline E2 level on the trigger day $(\mathrm{pg} / \mathrm{ml})$ & $201.7 \pm 15.24$ & $287 \pm 18.62$ & $279 \pm 18.21$ & $0.000 *$ \\
\hline Progesterone level day 8 post HCG injection & $8.75 \pm 1.76$ & $12.35 \pm 2.99$ & $13.28 \pm 3.3$ & 0.000 \\
\hline
\end{tabular}

*Statistically significant $(p<0.05)$

The Co- enzyme Q10 group (Group C) show better results than the L-Carnitine group (Group B) regarding rhe mean number of dominant follicles $>17 \mathrm{~mm}$, mean follicle size $(\mathrm{mm})$ on the day of trigger, mean endometrial thickness on the trigger day and progesterone level day 8 post HCG injection.

The cumulative ovulation rate (calculated after 3 months of induction) was significantly higher among the groups B and C compared to the control group A $(62.5 \%$ and $66.6 \%$ vs $18.3 \%$ respectively $\mathrm{p}$ value $<0.0001$ ). Overall pregnancy rate was significantly higher in study Groups as compared to control Group. Total number of cases who get pregnant after 3 month of treatment in Group B was 35(48.6\%), Group C 33(47.8\%) compared to Group A $6(8.5 \%)$.

The Clinical pregnancy was confirmed in $2(2.7 \%)$ women in Group A, in 33(44.6\%) in Group B and $32(43.2 \%)$ in Group $\mathrm{C}, \mathrm{p}$ value $<0.001$. There was significant difference in miscarriage rate between women in groups B, C (2.7\% each) compared to $(6.7 \%)$ in Group A. One patient had first trimester abortion and four cases were biochemical pregnancies in Group A, while in Group B no patients had first trimester abortion and only two cases were biochemical pregnancy, Also two cases aborted in Group $\mathrm{C}$ (one biochemical and one missed at 8 weeks of pregnancy). There was no reported cases of ectopic pregnancy in all groups. Three cases in group B and four cases in group $\mathrm{C}$ had twin pregnancy. There were no multiple pregnancies among pregnant women in group A. (Table 3). The L-Carnitine group (Group B) show slightly better results than the Co- enzyme Q10 group (Group C) regarding pregnancy rate. However they have the same results regarding the abortion rate.

Table 3: The outcomes of the study in study groups:

\begin{tabular}{|c|c|c|c|c|}
\hline \multirow{2}{*}{ Parameter } & Group (A) & Group (B) & Group (C) & \multirow{2}{*}{$P$-value } \\
\hline & $(\mathrm{n}=71)$ & $(\mathrm{n}=72)$ & $(\mathrm{n}=69)$ & \\
\hline Ovulation Rate (\% within Groups) & $13(18.3 \%)$ & $45(62.5 \%)$ & $46(66.6 \%)$ & $<0.0001 *$ \\
\hline $\begin{array}{l}\text { No. of pregnant cases ( total no. } 74 \text { ) } \\
\% \text { within Groups }\end{array}$ & $\begin{array}{c}6(8.1 \%) \\
6 / 71(8.5 \%)\end{array}$ & $\begin{array}{c}35(47.3 \%) \\
35 / 72(48.6 \%)\end{array}$ & $\begin{array}{l}33(44.6 \%) \\
33 / 69(47.8)\end{array}$ & $<0.0001^{*}$ \\
\hline $\begin{array}{l}\text { Clincal Pregnancy Rate (total no. } 67 \text { ) } \\
\% \text { within Pregnant cases }\end{array}$ & $\begin{array}{l}2(3 \%) \\
2.7 \%\end{array}$ & $\begin{array}{c}33(49.3 \%) \\
44.6 \%\end{array}$ & $\begin{array}{l}32(47.8 \%) \\
43.2 \%\end{array}$ & $<0.0001^{*}$ \\
\hline $\begin{array}{l}\text { Abortion rate (total no. } 10 \text { ) } \\
\% \text { within Pregnant cases }\end{array}$ & $\begin{array}{c}5(50 \%) \\
6.7 \%\end{array}$ & $\begin{array}{c}2(20 \%) \\
2.7 \%\end{array}$ & $\begin{array}{c}2(20 \%) \\
2.7 \%\end{array}$ & $<0.001^{*}$ \\
\hline $\begin{array}{l}\text { Multiple pregnancy } \\
\text { (\% within preg. no in each group) }\end{array}$ & $\begin{array}{l}0 / 6 \\
0 \%\end{array}$ & $\begin{array}{l}3 / 35 \\
8.6 \%\end{array}$ & $\begin{array}{c}4 / 33 \\
12.1 \%\end{array}$ & 0.627 \\
\hline
\end{tabular}

*Statistically significant $(p<0.05)$ 
Treatment continued and clomiphene given for 3 successive cycles for the cases who don't get pregnant and the data of each month were recorded. Analysis of the data and comparisons between groups every month regarding ovulation rate and pregnancy rate were done. The ovulation rate and pregnancy rate were maximum after the second month of ovulation induction $(11 \%, 41 \%, 38 \%$, ovulation rate in Group A, Group B, Group C respectively) with highly significant $p$ value between the control and study groups $<0.0001$ (Table 4 ).
While, the pregnancy rate was $(4.3 \%, 25.4 \%, 26.6 \%$ in Group A, Group B, Group C respectively) with significant $p$ value between the control and study groups 0.001 (Table 5).

L-Carnitine supplementation not only improved the ovulation and pregnancy rates, but also improved the Body Mass Index (BMI) when rechecked at the end of the study. BMI was significantly lower after treatment with L-Carnitine in Group B, p value 0.0039. (Table 6).

Table 4: The ovulation rate among the study groups over 3 month of treatment.

\begin{tabular}{lccccc}
\hline & Group A & Group B & Group C & Overall Ovulation rate & $P$ value \\
\hline \multirow{2}{*}{ st month } & $3(4 \%)$ & $10(13.3 \%)$ & $12(16 \%)$ & 25 & $0.049^{*}$ \\
2nd month & $8(11 \%)$ & $26(41 \%)$ & $24(38 \%)$ & 58 & $0.0001^{*}$ \\
3 3rd month & $2(3 \%)$ & $10(21.3 \%)$ & $10(22.7 \%)$ & 22 & $0.003^{*}$ \\
\hline
\end{tabular}

Table 5.: The pregnancy rate among the study groups over 3 month of treatment.

\begin{tabular}{|c|c|c|c|c|c|}
\hline & Group A & Group B & Group C & Overall Ovulation rate & $P$ value \\
\hline 1 st month & $1(1.3 \%)$ & $9(12 \%)$ & $8(10.7 \%)$ & 18 & $0.032 *$ \\
\hline 2nd month & $3(4.3 \%)$ & $16(25.4 \%)$ & $17(26.6 \%)$ & 36 & $0.001 *$ \\
\hline 3rd month & $2(3 \%)$ & $10(21.3 \%)$ & $8(18.2 \%)$ & 20 & $0.007 *$ \\
\hline
\end{tabular}

Table 6: The BMI among the study groups pre- and post -treatment.

\begin{tabular}{lccc}
\hline & BMI & BMI & $P$ value \\
\cline { 2 - 4 } & Pre-treatment & Post-treatment & 0.865 \\
\hline Group A $(\mathrm{n}=71)$ & $25.6 \pm 3.45$ & $25.7 \pm 3.56$ & $0.0039^{*}$ \\
Group B $(\mathrm{n}=72)$ & $25.7 \pm 3.36$ & $24.3 \pm 2.25$ & 0.626 \\
Group C $(\mathrm{n}=69)$ & $24.5 \pm 3.68$ & $24.2 \pm 3.54$ & 0.56 \\
\hline
\end{tabular}

\section{DISCUSSION}

Polycystic ovary syndrome (PCOS) is the most form of WHO type II anovulatory infertility and is associated with hyperandrogenemia. Moreover, PCOS is the most common endocrine disorder among females during reproductive age $^{[14]}$. PCOS not only a common cause of ovarian infertility but also considered as a systemic metabolic disorder, since the main pathophysiological mechanisms underlying this syndrome include obesity, insulin resistance and hyperinsulinemia. The impact of that syndrome on human reproduction include anovulatory infertility in addition to detrimental effects on oocyte, embryo quality endometrial receptivity and pregnancy development ${ }^{[3]}$.

Clomiphene citrate (CC) is still holding its place as the first-line therapy for ovulation induction in PCOS women. The most effective dosage is $100-150 \mathrm{mg} /$ day 
and up to $75 \%$ of ovulations occur within these doses. For those patients who fail to ovulate/conceive after at least 3 cycles of treatment with usual doses of CC (clomiphene resistance), other treatment modalities are needed ${ }^{[16]}$.

L-carnitine is reported to have beneficial effect in the treatment of infertility and as it is reported to be used as a potent antioxidant with little side effects and the researchers are now considering its implementation as a treatment for female infertility. Also, Coenzyme Q10 which is a component of the electron transport chain responsible for generating energy and being lipid-soluble antioxidant that could reduce oxidation stress within the ovary therefore enhancing female fertility and improving ovulation espicially in $\mathrm{PCO}$ women $^{[23]}$. So, throughout the current study, we aimed to assess the effectiveness of L-carnitine and CO- enzyme Q10 on the occurrence of ovulation in clomiphene resistant PCO infertile women because of their promising results from the literature and previous studies.

This study included 225 patients with PCOS between the age of 20 and 35 years. The diagnosis of PCOS was based on the 2003, Rotterdam criteria for diagnosis of PCOS with absent any other cause of infertility. They were randomly allocated into 3 groups each group included 75 patients but some cases withdrawn from the study and 212 patients continued till the end of study. Group A (72) received $150 \mathrm{mg}$ /day Clomiphene Citrate (CC) only, Group B received CC $150 \mathrm{mg} /$ day plus oral L-carnitine ( $2 \mathrm{~g}$ ) daily, Group C receive CC 150mg/day plus Co- enzyme Q10 (200 mg) daily.

All subjects received oral $\mathrm{CC}$ from day three until day seven of the cycle. Both L-carnitine and Co-enzyme Q10 taken from day three until the day of the first positive pregnancy test. Treatment continued for three successive cycles if pregnancy was not achieved.

No statistical difference between study groups regarding the baseline characteristics such as age, duration of infertility, type of infertility, body mass index and basal hormonal levels. The mean age of the patients among the groups was $27.6 \pm 4.6$ years while the mean BMI was $25.3 \pm 3.5$. Our data showed that the mean duration of infertility 3.93 of all groups. The majority of cases had primary infertility $48 / 64 \%, 52 / 69.3 \%, 46 / 61.3 \%$ in group A, B, C respectively.

It was found that the cumulative ovulation rate was much higher in cases received LCarnitine and Co-enzyme Q10 compared to women treated with CC only $(62.5 \%$ and $66.6 \%$ vs $18.3 \%$ respectively $p$ value $<0.0001$ ). Also the overall pregnancy was significantly higher in study Groups as compared to control Group. Total number of cases who get pregnant after 3 month of treatment in Group B was 35(48.6\%), Group C 33(47.8\%) compared to Group A 6(8.5\%). Adding L-Carnitine and Co-enzyme Q10 to CC improved not only the quantity but also the quality of ovulation proved by increased clinical pregnancy rate in
Group B 33(44.6\%) and Group C 32(43.2\%) compared to Group A 2(2.7\%) $p$ value $<0.000$.

This was similar to study of AM. Ismail et al. who showed that the combination of L-carnitine and clomiphene citrate significantly improved both the ovulation and the pregnancy rates in clomiphene resistant PCOS $(64.7 \%$ vs. $17.6 \%$ and $51.25 \%$ vs. $5.8 \%)^{[22]}$. AM. Ismail et al. studied 170 clomiphene resistant PCOS women (aged less than 35 years) that were randomly allocated into 2 groups only: Group A $(\mathrm{n}=85)$ received $250 \mathrm{mg}$ clomiphene citrate plus L-Carnitine, Group B $(\mathrm{n}=85)$ received 250 $\mathrm{mg}$ clomiphene citrate with placebo. They found that the number of stimulated follicles reaching $\geq 17 \mathrm{~mm}$ diameter was significantly higher in Group A as compared to Group B ( $2.21 \pm 0.1$ vs $0.16 \pm 0.7)$, similarly the endometrial thickness at the time of hCG administration was significantly thicker in Group A $(10.1 \pm 0.1 \mathrm{~mm})$ vs. $(6.8 \pm 0.4 \mathrm{~mm})$ in Group B, $p$-value (0.0001). This may be related to higher Serum E2 on the day of hCG injection due to better ovulation.

These results came along with our study in which the endometrial thickness (ET) obtained on day of hCG trigger was significantly higher in Group B with mean ET $8.5 \pm 0.76$, and in Group $C$ with mean $8.8 \pm 0.87$ compared to group A mean ET $6.1 \pm 0.71(P=0.000)$. Also a highly significant difference in the mean number of follicles $>17 \mathrm{~mm}$ was detected between Group A $(0.21 \pm 0.47)$ compared to Goup B $(0.93 \pm 0.89)$ and Group C (1.19 \pm 1.06$)$. The follicle size was signficantly larger in Group B and C compared to Group A (18.18, 18.45 vs 17.34 respectively, $p$ value $(<0.0001)$. E2 level on day of hCG trigger and progesterone level on day 8 post hCG noticed to be higher in Group C and Group B than in Group A. Even hCG injection was given earlier in Group C and Group B compared to Group A because of rapid follicular growth and maturatuion. The mean day of hCG trigger was $15.25 \pm 1.13,11.37 \pm 1.08,10.93 \pm 1.18$ in Group $\mathrm{A}, \mathrm{B}, \mathrm{C}$ respectively.

Our study differs than AM. Ismail. who tested the addition of L-Carnitine to clomiphene citrate in clomiphene-resistant PCOS, While our study tested the addition of co-enzyme Q10 to CC vs. 1-carnitine to CC in clomiphene-resistant PCOS.

The results of this work came consistent to that of El Refaeey A. et al. who studied a total of 101 infertile women with PCOS that were randomized either to combined CoQ10 and clomiphene citrate or to clomiphene citrate alone, Ovulation occurred in $65.9 \%$ in the CoQ10 group compared with only $15.5 \%$ in the control group. Pregnancy rate was significantly higher in the CoQ10 group (37.3\%) versus the control group (6.0\%). Cases who were supplemented with CoQ10 show more follicles $>17 \mathrm{~mm}$ and greater endometrial thickness ${ }^{[27]}$.

After 3 months of treatment, the improvement in pregnancy and ovulation rates were comparable between women treated with $\mathrm{CC}$ - only and those women given 
either L-Carnitine plus CC or Co-enzyme Q10 plus CC. The ovulation rate and pregnancy rate were maximum after the second month of ovulation induction $(11 \%, 41 \%, 38 \%$, ovulation rate in Group A, Group B, Group C respectively) with highly significant $\mathrm{p}$ value between the control and study groups $<0.0001$, while the pregnancy rate was $(4.3 \%, 25.4 \%, 26.6 \%$ in Group A, Group B, Group C respectively) with significant $\mathrm{p}$ value between the control and study groups 0.001 . This means that treatment with both L-Carnitine and Co-enzyme Q10 in PCOS women is maximally effective after two month of treatment.

The current study came along with study done by Latifian et al that tested the effect of adding L-Carnitine to PCOS women who were resistant to clomiphene citrate and gonadotropin. L-Carnitine given from the third day of treatment with clomiphene citrate and gonadotropin in a dose $2 \mathrm{gm}$ oral every $12 \mathrm{~h}$ until the hCG injection. Their study included 50 PCOS patients (aged 20-35 years) with complaints of infertility and ovulatory disorders that received stimulation with Clomiphene Citrate and Gonadotropins for at least two cycles but no dominant follicles, were selected, and the effects of adding L-Carnitine on follicular growth rate and fertility have been examined in the next cycle. Research results revealed that women supplemented with LC had a considerable change in the response of ovaries with increased follicle size (mainly left ovary) compared to those without LC. With the administration of L-Carnitine the dominant follicles were observed in the ovaries of $64 \%$ of patients and $20 \%$ of the patient had positive pregnancy tests, also the endometrium thickness was significantly higher after LC supplementation(28).

The results of the current work may impose that L-carnitine and Co-enzyme Q10 not only helps in improving the results of ovulation induction and conception but also assist in early pregnancy success evidenced by the low abortion rate (biochemical and first trimestric abortions) in Groups B and C compared to Group A $(2.7 \%$ vs. $6.7 \%)$. One patient had first trimester abortion and four cases were biochemical pregnancies in Group A, while in Group B no patients had first trimester abortion and only two cases were biochemical pregnancy, Also two cases aborted in Group C(one biochemical and one missed at 8 weeks of pregnancy). This meets with the results of AM. Ismail et al study in which the miscarriage rate was lower in L-Carnitine group $(2 / 85 ; 2.3 \%)$ than the control group $(4 / 85 ; 4.7 \%)(\mathrm{p}=0.67)$.

The previous observation of L-carnitine and Co-enzyme Q10 that they help in early pregnancy success through increasing the clinical pregnancy rate might be explained first by: increase in endometrial receptivity because of the increase in the mean endometrial thickness at the day of HCG administration throughout the study, second by: good quality of the follicles and developing embryos which is attributed to the antioxidant effect of CoQ10 and LC in improving egg quality and ovulation by repairing free radicals ${ }^{[20,24]}$.

Three cases in group B and four cases in group C had twin pregnancy. There were no multiple pregnancies among pregnant women in group A.

L-Carnitine supplementation not only improved reproductive outcome, but also enhanced the patients' weight and Body Mass Index (BMI). Samimi et al, found that LC supplementation $(250 \mathrm{mg}$ per day orally for 12 weeks) lead to significant reduction in body BMI, weight, waist and hip circumference ${ }^{[25]}$. Also, in the present study L-Carnitine supplementation improved the ovulation, pregnancy rates and the Body Mass Index (BMI) of the studied group. BMI was significantly lower after treatment with L-Carnitine in Group B (pre-treatment BMI 25.7 \pm 3.36 , post-treatment BMI $24.3 \pm 2.25)$ $p$ value 0.0039 .

Pregnancy rates are the name of the game in women taking the common conventional agent used in this study and the results in this study were compelling in this regard. Explanations for this can include:

-Direct action of L C on the oocytes as it increases energy production in mitochondria and maintaining acetyl $\mathrm{CoA} / \mathrm{CoA}$ ratio.

- It also reduces oxidative stress and lipotoxicity via the free radical scavenging effect with subsequent effects on the endometrial blood flow ultimately supporting healthy endometrial receptivity in the peri-implantation phase.

- It promotes oocyte growth and maturation by decreasing the rate of apoptosis.

\section{CONCLUSION}

Both L-carnitine and Co-enzyme Q10 were effective with promising results in improving pregnancy and ovulation rates for women with CC-resistant PCOS. However L-carnitine was superior in improving BMI in the study group.

L-carnitine and CoQ10 reach their maximum effect after two month of treatment unlike metformin, which requires up to 6 months to produce its proper effect, also they are not as expensive as gonadotrophins and does not need intensive monitoring during and after treatment and was well tolerated by most of the patients with very mild adverse effects were observed in few patients (very mild nausea and vomiting at the start of treatment and stopped after continuing the treatment).

The results of this study are encouraging; however, the appropriate dosage and duration of treatment needs to be further investigated.

The present study is the first to compare the reproductive effects of L-carnitine versus Co-enzyme Q10 therapy for 
women with PCOS, further studies are needed to confirm their benfit as an adjuvant therapy during induction of ovulation in PCOS women especially those who are resistant to the conventional methods of treatment.

\section{REFERENCES}

1. Khaduri MA, Faris YA, Najjar TA, Gowri V. Hospital - based prevelance of polycystic ovarian syndrome among Omani women. Middle East Fertil Soc J 2014; 19; 135-8

2. Rashidi H, Ramezani Tahrani F, Bahri Khomami M, Tohidi M, Azizi F. To what extent does the use of the Rotterdam criteria affect the prevelance of polycystic ovary syndrome? A community- based study from the Southwest of Iran.Eur J Obstet Gynecol Repod Biol $2014 ; 174: 100-5$.

3. Hart R and Doherty DA. The potential implications of PCO diagnosis on a woman long term health. $\mathrm{J}$ of Clinical Endocrinology and Metabolism 2015; 100.3:911-919

4. Berek J. Novaks gynecology 13ed. Philadelphia:Lippincott Williams and Wilkins.2002.

5. ESHRE Capri Workshop Group Anovulatory infertility.Human Reproduction.1995; Report No.10

6. Arredondo F, Noble LS. Endocrinology of recurrent pregnancy loss. Semin Reprod Med 2006;24: 33 -9.

7. Balen AH, Tan SL, MacDougall J, JACOBS HS. Miscarriage rates following in vitro fertilization are increased in women with polycystic ovaries and reduced by pituitary desensitization with buserelin. Hum Reprod 1993; 8: 959-64.

8. Martens JW, Geller DH, Arlt W, AuchusRJ, Ossovskaya VS, Rodriguez $\mathrm{H}$, et al. Enzymatic activities of P450c17 stably expressed in fibroblasts from patients with the polycystic ovarian syndrome. J Cline Endocrinol Metab.2000;85 (11), 4338-46

9. Speroff L, Fritz M. Clinical gynecoloic endocrinology and infertility. 7 ed, Philadelphia:Lippincott Williams and Wilkins 2005.

10. Ke RW. Endocrine basis for recurrent pregnancy loss. Obstet Gynecol Clin North Am.2014;41:103-12.

11. Miyamoto K, Sato EF, Kasahara E, et al. Effect of oxidative stress during repeated ovulation on the structure and functions of the ovary, oocytes, and their mitochondria. Free Radic Biol Med 2010;49(4)674-81 Epub.
12. Ringseis R, Eder K, Keller J. Role of carnitine in the regulation of glucose homeostasis and insulin sensitivity: evidence from in vivo and in vitro studies with carnitine supplementation and carnitine deficiency. Eur J Nutr 2012;51 (1):1-18.

13. Trivax B, Azziz R. Diagnosis of polycystic ovary syndrome. Clin Obstet Gynecol 2007;50(1):168-77.

14. Clark JH, Markaverich BM. The agonist-antagonist properties of clomiphene. Pharmacol Ther 1981;15:467-519.

15. Brown J, Farquhar C, Beck J, Boothroyd C, Hughes E.Clomiphene and anti-estrogens for ovulation induction in PCOS. Cochrane Databaseof Systematic Reviews. 2009; Issue 4. Art. No. CD002249.

16. Ahmed Rizk AY, Mohamed Bedaiwy MA, Hesham Al-Inany HG. N-acetyl-cysteine is a novel adjuvant to clomiphene citrate in clomiphene citrateresistant patients with polycystic ovary. Fertil Steril 2005; 83(2):367-70

17. Wu GQ, Jia BY, Li JJ, Fu XW, Zhou GB , Hou YP, Zhu SE. L-carnitine enhances ocyte maturation and developmentrt of parthenogenetic embryos in pigs. Theriogenology .2011; Sep:15:76(5):785-93

18. Abdelrazik H, Sharma R, Mahfouz R, Agarwal A. L-Carnitine decreases DNA damage and improves the in vitro blastocyst development rate in mouse embryos. Fertil Steril 2008;91(2)589-96 Published online.

19. Wall BT, Stephens FB, Constantin-Teodosiu D, Marimuthu K, Macdonald IA, Greenhaff PL. Chronic L-carnitine oral ingestion and carbohydrate increase muscle fuel metabolism. J Physiol 2011; Feb 15;589(4):963-73.

20. Pillich RT, Scarsella G, Risuleo G: Reduction of apoptosis through the mitochondrial pathway by the administration of acetyl-1-carnitine to mouse fibroblasts in culture. Exp Cell Res. 2005, 306(1):1-8.

21. Fenkci SM, Fenkci V, Oztekin O, Rota S, Karagenc N. Serum total L-carnitine levels in non-obese women with polycystic ovary syndrome. Hum Reprod. 2008; 23: 1602-1606.

22. Alaa M. Ismail, Ali Hassan Hamed, Srdjan Saso, Hossam H. Thabet. Adding L-Carnitine to clomiphene resistant PCOS women improves the quality of ovulation and pregnancy rate. Eur J Obstet Gynecol Reprod Biol 2014; Sep;180:148-152.

23. Burstein E, Perumalsamy A, Bentov Y, Esfandiari N, Jurisicova A, Casper RF. Co-enzyme q10 
supplementation improves ovarian response and mitochondrial function in aged mice [abstract]. Fertil Steril. 2009;92(Suppl):S31.

24. Bentov Y, Casper RF. The aging oocyte-can mitochondrial function be improved? Fertil Steril. 2013; Jan;99(1):18-22.

25. Samimi, M., Zarezade Mehrizi, M., Foroozanfard, F., et al. The effects of coenzyme Q10 supplementation on glucose metabolism and lipid profiles in women with polycystic ovary syndrome: a randomized, doubleblind, placebo-controlled trial. Clin Endocrinol.2017.

26. Quinzii, C.M., Hirano, M., DiMauro, S. CoQ10 deficiency diseases in adults. Mitochondrion.2007; 7, S122-S126.

27. El Refaeey A, Selem A, Badawy A.Combined coenzyme Q10 and clomiphene citrate for ovulation induction in clomiphene-citrate-resistant polycystic ovary syndrome.Reprod Biomed Online. 2014; Jul;29(1):119-24.

28. Latifian S, Hamdi K, Totakneh REffect of addition of l-carnitine in polycystic ovary syndrome (PCOS) patients with clomiphene citrate and gonadotropin resistant. Int J Curr Res Acad Rev.2015; 3:469-76. 